International Journal of Pure and Applied Mathematics

Volume 110 No. 2 2016, 311-326

ISSN: 1311-8080 (printed version); ISSN: 1314-3395 (on-line version)

url: http://www.ijpam.eu

doi: 10.12732 /ijpam.v110i2.6

\title{
A STUDY ON TEMPERATURE-DISTRIBUTION AND FIN EFFICIENCY OF CONVECTIVE STRAIGHT FINS WITH TEMPERATURE DEPENDENT THERMAL CONDUCTIVITY \\ OF FRACTIONAL ORDER ENERGY BALANCE EQUATION BY USING ADOMIAN DECOMPOSITION SUMUDU TRANSFORM METHOD
}

\author{
Trushit Patel ${ }^{1}$, Ramakanta Meher $^{2} \S$ \\ ${ }^{1,2}$ Applied Mathematics and Humanities Department \\ S.V. National Institute of Technology \\ Surat, 395007, INDIA
}

\begin{abstract}
In this study, an attempt has been made to fractionalize the governing energy balance equation and Adomian decomposition Sumudu transform method (ADSTM) is used to solve the nonlinear fractional order energy balance equation to find the temperature distribution and to check the efficiency of convective straight fin with temperature dependent thermal conductivity. The thermal conductivity of the fin material is assumed as a linear function of temperature to find the temperature distribution for different values of thermal conductivity and thermo-geometric fin parameters for fractional as well as for integer order energy balance equation. Finally, the obtained ADSTM results has been compared with Variational Iteration Method (VIM) (Coskun et al., 2008) and Numerical method to check the accuracy of the purposed method.
\end{abstract}

AMS Subject Classification: 34A08, 34B15, 49K15, 45J05

Key Words: Adomian Decomposition Sumudu Transform Method, Fractional Order Energy Balance Equation, Convective straight fins, Thermal conductivity, Temperature distribution, Fin efficiency

Received: June 25, 2016

Revised: $\quad$ September 16, 2016

Published: $\quad$ November 4, 2016

${ }^{\S}$ Correspondence author (c) 2016 Academic Publications, Ltd. url: www.acadpubl.eu 


\section{Introduction}

Fins are generally employed to enhance the heat transfer between the primary surface and its convective, radiating or convective-radiating environment. Now a days extended surfaces (fins) are widely used in many engineering applications like refrigeration, air conditioning, chemical processing and automobile equipment. Kraus et al. [1] discussed number of cases of the energy balance equation and allowed closed form analytical solutions by assuming thermophysical properties as constant having uniform heat transfer coefficient which reduces the mathematical complexity.

Numerous contributions has been made so far in the heat transfer analysis of the fins. Razelos et al. [2] considered the variation of the convective heat transfer coefficient from the base of a convecting fin to its tip. A simplified solution for the efficiency and optimization of fin with temperature-dependent thermal conductivity was studied by Bouaziz et al. [3]. Cihat [4] used Adomian Decomposition Method and Rajabi [5] used Homotopy Perturbation method to find the fin efficiency of convective straight fins with temperature-dependent thermal conductivity and to determine the temperature distribution within the fin. Coskun et al. [6] used Variational iteration method to study the analysis of convective straight and radial fins with temperature-dependent thermal conductivity and compared their results with finite element analysis. Similarly, Ganji et al. [7] applied Homotopy Perturbation Method to study the fin efficiency and temperature distribution with temperature-dependent thermal conductivity in convective straight fins.

In the last two decades, fractional calculus starts to intervene significantly in engineering, physics, economics etc. It is due to new possibilities in which fractional calculus brings into the modelling of various problems. In recent years, the fractional differential equations gained much attention because of its numerous applications in science and engineering. Some important applications are fluid flows, heat transfer, material science, signal processing and control theory. During the last three decades, the fractional calculus has found its applications in several apparently diverse fields of science and engineering (see [8], [9], [10] [11], [12], [13], and [14]). Besides the recent developments in traditional numerical methods and analytical methods for solving fractional order differential equation, some novel approaches such as Adomian decomposition Sumudu transform method has been presented here to deals with the fractional order differential equation with less or strong nonlinearity. The ADSTM is a combination of Sumudu transform and Adomian decomposition method. In this paper a straight fin with temperature dependent thermal conductivity is 
considered and an attempt has been made to fractionalize the governing energy balance equation to study the anomalous behavior of the system and Adomian decomposition Sumudu transform method is used to solve the fractional order energy balance to find the fin temperature and to study the temperature distribution in a straight fin for different values of thermal conductivity and thermo-geometric fin parameters.

\section{Sumudu Transform}

In the early 90's, Watugala [15] introduced a new integral transform, named the Sumudu transform and applied it to find the solution of ordinary differential equation in control engineering problems. Consider the set of functions

$$
A=\left\{f(t)\left|\exists M, \tau_{1}, \tau_{2}>0, \quad\right| f(t) \mid<M e^{\frac{|t|}{\tau_{j}}}, \quad \text { if } t \in(-1)^{j} \times[0, \infty)\right\},
$$

then, Sumudu transform of $f(t)$ be defined as follows [16]

$$
F(u)=S[f(t)]=\int_{0}^{\infty} f(u t) e^{-t} d t, \quad u \in\left(-\tau_{1}, \tau_{2}\right)
$$

If $F(u)$ be the Sumudu Transform of $f(t)$, then Sumudu transform of the Caputo fractional derivative is given as follows [17]

$$
S\left[D_{t}^{\alpha} f(t)\right]=u^{-\alpha} S[f(t)]-\sum_{k=0}^{m-1} u^{-\alpha+k} f^{(k)}(0+), \quad m-1<\alpha \leq m .
$$

Some fundamental properties of Sumudu Transform were established by Asiru [17], and Belgacem [18]. Recently, this transform is applied to solve the system of differential equations and partial differential equations; see Kilicman et al. in [19] and [20].

\section{Basic Ideas of Adomian Decomposition Sumudu Transform Method (ADSTM)}

Consider a fractional order nonlinear nonhomogeneous differential equation as

$$
D_{t}^{\alpha} \theta(\zeta)+R \theta(\zeta)+N \theta(\zeta)=g(\zeta)
$$


with initial condition

$$
\theta(0)=\theta_{0}
$$

where $D_{\zeta}^{\alpha}$ is the Caputo fractional derivative of the function $\theta(\zeta), R$ is reminder term or linear differential operator, $N$ represents the general nonlinear differential operator, and $g(\zeta)$ is the source term.

Taking Sumudu Transform (denoted by $S$ ) on both sides of equation (4), it obtain

$$
S\left[D_{\zeta}^{\alpha} \theta(\zeta)\right]+S[R \theta(\zeta)]+S[N \theta(\zeta)]=S[g(\zeta)]
$$

By using the property of Sumudu transform method and applying inverse Sumudu transform in equation (6), it gives

$$
\theta(\zeta)=G(\zeta)-S^{-1}\left[u^{\alpha} S[R \theta(\zeta)+N \theta(\zeta)]\right]
$$

where $G(\zeta)=S^{-1}\left[\theta(0)+u^{\alpha} S[g(\zeta)]\right]$ represents the term arising from the source term and the prescribed initial conditions.

The approximate solution of equation (7) can be written in the form

$$
\theta(\zeta)=\sum_{n=0}^{\infty} \lambda^{n} \theta_{n}(\zeta)
$$

and the nonlinear terms present in equation (7) can be expressed as a sum of Adomian polynomials

$$
N \theta(\zeta)=\sum_{n=0}^{\infty} \lambda^{n} A_{n}(\theta)
$$

where the Adomian polynomials $A_{n}(\theta)$ can be defined as [21]

$$
A_{n}\left(\theta_{0}, \theta_{1}, \theta_{2}, \ldots, \theta_{n}\right)=\frac{1}{n !} \frac{\partial^{n}}{\partial \lambda^{n}}\left[N\left(\sum_{i=0}^{\infty} \lambda^{i} \theta_{i}\right)\right]_{\lambda=0}, \text { for } n=0,1,2, \ldots
$$

Substituting Eqs. (8) and (9) in equation (7) and applying the inverse Sumudu Transform, we find

$$
\sum_{n=0}^{\infty} \lambda^{n} \theta_{n}(\zeta)=G(\zeta)-\lambda\left[S^{-1}\left[u^{\alpha} S\left[R \sum_{n=0}^{\infty} \lambda^{n} \theta_{n}(\zeta)+\sum_{n=0}^{\infty} \lambda^{n} A_{n}(\theta)\right]\right]\right]
$$

The resulting equation (11) is the coupling of the Sumudu Transform and the Adomian Decomposition Method. 
Now by equating the coefficients of like powers of $\lambda$, in equation (11), the following iterated terms can be obtained as

$$
\begin{aligned}
& \lambda^{0}: \theta_{0}(\zeta)=G(\zeta) \\
& \lambda^{1}: \theta_{1}(\zeta)=-S^{-1}\left[u^{\alpha} S\left[R \theta_{0}(\zeta)+A_{0}(\theta)\right]\right] \\
& \lambda^{2}: \theta_{2}(\zeta)=-S^{-1}\left[u^{\alpha} S\left[R \theta_{1}(\zeta)+A_{1}(\theta)\right]\right] \\
& \lambda^{3}: \theta_{3}(\zeta)=-S^{-1}\left[u^{\alpha} S\left[R \theta_{2}(\zeta)+A_{2}(\theta)\right]\right]
\end{aligned}
$$

By considering this above procedure, $\theta_{n}(\zeta)$ can be completely obtained implies the series solution can be determined subsequently and hence the resulted approximate analytical solution $\sum_{n=0}^{M} \theta_{n}(\zeta)$ converges to the exact solution $\theta(\zeta)$ as $M \rightarrow \infty$.

\section{Mathematical Model}

Consider a fin as shown in Figure 1 which represents a straight fin with temperaturedependent thermal conductivity. The cross sectional area $A_{c}$ having perimeter $P$ is constant and fin length is $b$. The fin is attached to a base surface of temperature $T_{b}$ and extends it into a fluid of temperature $T_{a}$ keeping its tip be insulated.

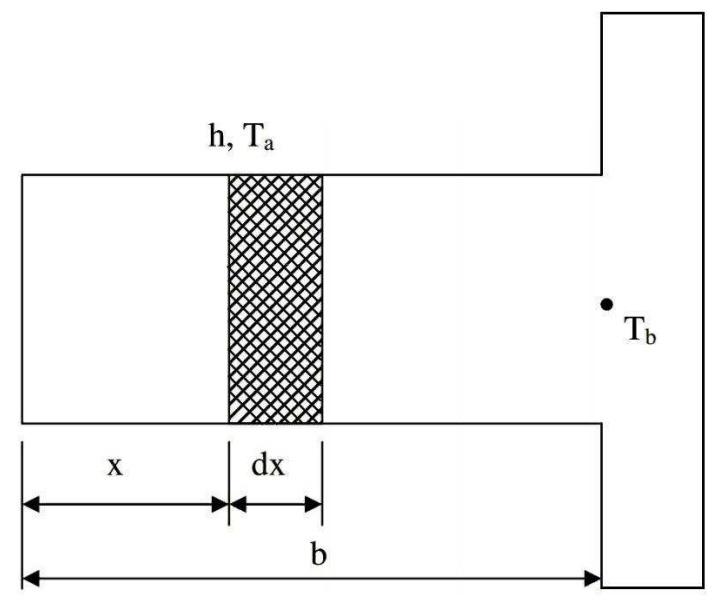

Figure 1: Geometry of a straight fin 
The energy balance equation for a differential element of the fin be given by

$$
A_{c} \frac{d}{d x}\left[k(T) \frac{d T}{d x}\right]-P h\left(T_{b}-T_{a}\right)=0
$$

Here, the thermal conductivity of the fin material is assumed to be a linear function of temperature, so according to (see [4], [5], [6], and [7])

$$
k(T)=\lambda_{a}\left[1+\tilde{\lambda}\left(T-T_{b}\right)\right]
$$

where $\lambda_{a}$ is the thermal conductivity at the ambient fluid temperature of the fin and parameter $\tilde{\lambda}$ describing the slope of the thermal conductivity.

With the introduction of the following dimensionless quantities

$$
\xi=\frac{x}{b}, \quad \theta=\frac{T-T_{a}}{T_{b}-T_{a}}, \quad \psi=\left(\frac{h P b^{2}}{\lambda_{a} A_{c}}\right)^{\frac{1}{2}} \quad \text { and } \beta=\tilde{\lambda}\left(T_{b}-T_{a}\right)
$$

equation (13) reduces to the following form

$$
\frac{d^{2} \theta}{d \zeta^{2}}+\beta \theta \frac{d^{2} \theta}{d \zeta^{2}}+\beta\left(\frac{d \theta}{d \zeta}\right)^{2}-\psi^{2} \theta=0 ; \quad 0 \leq \zeta \leq 1
$$

The corresponding dimensionless boundary conditions are

$$
\theta(\zeta=1)=1 \text { and } \theta^{\prime}(\zeta=0)=0
$$

Implies the computational domain be transformed to $0 \leq \zeta \leq 1$ with the introduction of the dimensionless parameters as given in equation (15).

\section{Application of ADSTM for Finding fin Temperature in Fractional Order Energy Balance Equation}

To understand the anomalous behavior of this system we fractionalize the energy balance equation (16) into fractional order $(\alpha>0)$ in order to study the behavior of temperature distribution in straight fins

$$
\frac{d^{\alpha} \theta}{d \zeta^{\alpha}}+\beta \theta \frac{d^{2} \theta}{d \zeta^{2}}+\beta\left(\frac{d \theta}{d \zeta}\right)^{2}-\psi^{2} \theta=0 ; \quad 1<\alpha \leq 2 \text { and } 0 \leq \zeta \leq 1
$$

with appropriate boundary conditions (17). 
equation (18) can be written as

$$
\frac{d^{\alpha} \theta}{d \zeta^{\alpha}}=\psi^{2} \theta-\beta\left[\theta \frac{d^{2} \theta}{d \zeta^{2}}+\left(\frac{d \theta}{d \zeta}\right)^{2}\right]
$$

Now, applying sumudu transform and then inverse sumudu transform on both sides of equation (19), it obtains

$$
S[\theta(\zeta)]=K-S^{-1}\left[u^{\alpha} S\left[\beta\left[\theta \frac{d^{2} \theta}{d \zeta^{2}}+\left(\frac{d \theta}{d \zeta}\right)^{2}\right]-\psi^{2} \theta(\zeta)\right]\right]
$$

by applying Adomian Decomposition Method, it obtain the following equation

$$
\sum_{n=0}^{\infty} \lambda^{n} \theta_{n}(\zeta)=K-\lambda\left[S^{-1}\left[u^{\alpha} S\left[\beta\left[\sum_{n=0}^{\infty} \lambda^{n} A_{n}(\theta)\right]-\sum_{n=0}^{\infty} \lambda^{n} \psi^{2} \theta_{n}(\zeta)\right]\right]\right]=0
$$

where $A_{n}(\theta)$ is Adomian's polynomial which represents for the nonlinear terms and can be obtained by using equation (10).

The first few components of Adomian's polynomial for the corresponding nonlinear term is given by

$$
\begin{aligned}
& A_{0}=\theta_{0} \frac{d^{2} \theta_{0}}{d \zeta^{2}}+\left(\frac{d \theta_{0}}{d \zeta}\right)^{2} \\
& A_{1}=\theta_{1} \frac{d^{2} \theta_{0}}{d \zeta^{2}}+\theta_{0} \frac{d^{2} \theta_{1}}{d \zeta^{2}}+2 \frac{d \theta_{0}}{d \zeta} \frac{d \theta_{1}}{d \zeta} \\
& A_{2}=\theta_{2} \frac{d^{2} \theta_{0}}{d \zeta^{2}}+\theta_{1} \frac{d^{2} \theta_{1}}{d \zeta^{2}}+\theta_{0} \frac{d^{2} \theta_{2}}{d \zeta^{2}}+\left(\frac{d \theta_{1}}{d \zeta}\right)^{2}+2 \frac{d \theta_{0}}{d \zeta} \frac{d \theta_{2}}{d \zeta}
\end{aligned}
$$

On comparing the coefficients of like powers of $\lambda$ in equation (21), the iterated terms can be represented as

$$
\begin{aligned}
\theta_{0}(\zeta) & =K \\
\theta_{n+1}(\zeta) & =S^{-1}\left[u^{\alpha} S\left[\psi^{2} \theta_{n}(\zeta)-\beta A_{n}(\theta)\right]\right], \quad n=0,1,2 \ldots
\end{aligned}
$$

and it gives

$$
\begin{aligned}
& \theta_{0}(\zeta)=K \\
& \theta_{1}(\zeta)=-S^{-1}\left[u^{\alpha} S\left[\beta A_{0}(\theta)-\psi^{2} \theta_{0}(\zeta)\right]\right]
\end{aligned}
$$




$$
\begin{aligned}
& =\frac{K \psi^{2} \zeta^{\alpha}}{\Gamma(\alpha+1)} \\
\theta_{2}(\zeta) & =-S^{-1}\left[u^{\alpha} S\left[\beta A_{1}(\theta)-\psi^{2} \theta_{1}(\zeta)\right]\right] \\
& =\frac{K \psi^{4} \zeta^{2 \alpha}}{\Gamma(2 \alpha+1)}-\frac{K^{2} \psi^{2} \beta \zeta^{2 \alpha-2}}{\Gamma(2 \alpha-1)} \\
\theta_{3}(\zeta) & =-S^{-1}\left[u^{\alpha} S\left[\beta A_{2}(\theta)-\psi^{2} \theta_{2}(\zeta)\right]\right] \\
& =\frac{K^{3} \beta^{2} \psi^{2} \zeta^{3 \alpha-4}}{\Gamma(3 \alpha-3)}+\frac{K \psi^{6} \zeta^{3 \alpha}}{\Gamma(3 \alpha+1)}-\frac{K^{2} \psi^{4} \beta \alpha(\alpha-1) \Gamma(2 \alpha-1) \zeta^{3 \alpha-2}}{(\Gamma(\alpha+1))^{2} \Gamma(3 \alpha-1)} \\
& -\frac{K^{2} \psi^{4} \beta \zeta^{3 \alpha-2}}{\Gamma(3 \alpha-1)}-\frac{K^{2} \psi^{4} \beta \Gamma(2 \alpha-1) \zeta^{3 \alpha-2}}{(\Gamma(\alpha+1))^{2} \Gamma(3 \alpha-2)}-\frac{K^{2} \psi^{4} \beta \zeta^{3 \alpha-2}}{\Gamma(3 \alpha-1)}
\end{aligned}
$$

The approximate solution of equation (18) corrected upto 5 terms, can be expressed as

$$
\theta(\zeta)=\theta_{0}(\zeta)+\theta_{1}(\zeta)+\theta_{2}(\zeta)+\theta_{3}(\zeta)+\theta_{4}(\zeta)+\ldots
$$

Which represents an expression for fin temperature of fractional order energy balance equation, where the coefficient $K$ denotes the temperature at the fin tip, that can be obtained by using the boundary condition $\theta(\zeta=1)=1$ which must lies within the interval $[0,1]$.

\section{Stability Analysis via Fixed Point Theorem}

Let $(X,\|\|$.$) be a Banach space and H$ a self-map of $X$. Let $\theta_{n+1}=f\left(H, \theta_{n}\right)$ be a particular recursive procedure. Suppose $F(H)$ be a fixed point set of $H$ having at lease one element such that $\theta_{n}$ converges to a point $p \in F(H)$. Let $\theta_{n} \subseteq X$ and define $e_{n}=\left\|\theta_{n+1}-f\left(H, \theta_{n}\right)\right\|$. If $\lim _{n \rightarrow \infty} \theta^{n}=p$, then the iteration $\theta_{n+1}=f\left(H, \theta_{n}\right)$ is said to be $H$ - stable. Without any loss of generality, we must assume that, our sequence $\theta_{n}$ has an upper boundary; otherwise we cannot expect the possibility of convergence. If all these conditions are satisfied for $\theta_{n+1}=H \theta_{n}$ which is known as Picards iteration, then the iteration will be $H$-Stable. Now, we state the following theorem [22].

Theorem 1. Let $(X,\|\|)$ be a Banach space and $H$ a self-map of $X$ satisfying

$$
\left\|H_{x}-H_{y}\right\| \leq C\left\|x-H_{x}\right\|+c\|x-y\|,
$$

for all $x, y$ in $X$ where $0 \leq C .0 \leq c<1$. Then $H$ is Picard H-Stable. 
Let the following succession correlate to the nonlinear fractional order energy balance equation ( equation (23)),

$$
\theta_{n+1}(\zeta)=S^{-1}\left[u^{\alpha} S\left[\psi^{2} \theta_{n}(\zeta)-\frac{\beta}{2} \frac{d^{2}}{d \zeta^{2}}\left(\theta_{n}^{2}(\zeta)\right)\right]\right], n=0,1,2 \ldots
$$

Theorem 2. Let $T$ be a self-map defined as

$$
\begin{aligned}
T\left(\theta_{n}(\zeta)\right) & =\theta_{n+1}(\zeta) \\
& =S^{-1}\left[u^{\alpha} S\left[\psi^{2} \theta_{n}(\zeta)-\frac{\beta}{2} \frac{d^{2}}{d \zeta^{2}}\left(\theta_{n}^{2}(\zeta)\right)\right]\right]
\end{aligned}
$$

is $T$ - stable in $L^{2}(a, b)$ if $\left\{\beta_{1}-\beta_{2} \kappa\right\}<1$.

Proof. The first step of the proof will consist on showing that $T$ has a fixed point. To achieve this, we evaluate the following for all $(n, k) \in N \times N$

$$
\left\|T\left(\theta_{n}(\zeta)\right)-T\left(\theta_{k}(\zeta)\right)\right\|=\left\|\begin{array}{l}
S^{-1}\left[u^{\alpha} S\left[\psi^{2} \theta_{n}(\zeta)-\frac{\beta}{2} \frac{d^{2}}{d \zeta^{2}}\left(\theta_{n}^{2}(\zeta)\right)\right]\right] \\
-S^{-1}\left[u^{\alpha} S\left[\psi^{2} \theta_{k}(\zeta)-\frac{\beta}{2} \frac{d^{2}}{d \zeta^{2}}\left(\theta_{k}^{2}(\zeta)\right)\right]\right]
\end{array}\right\|
$$

Using the linearity property of the inverse sumudu transform, we obtain

$$
\left\|T\left(\theta_{n}(\zeta)\right)-T\left(\theta_{k}(\zeta)\right)\right\|=\left\|S^{-1}\left[u^{\alpha} S\left\{\begin{array}{c}
\psi^{2}\left(\left(\theta_{n}(\zeta)-\theta_{k}(\zeta)\right)\right. \\
-\frac{\beta}{2} \frac{d^{2}}{d \zeta^{2}}\left(\theta_{n}^{2}(\zeta)-\theta_{k}^{2}(\zeta)\right)
\end{array}\right\}\right]\right\|
$$

By using the triangle inequality for the norm, we get

$$
\begin{aligned}
\left\|T\left(\theta_{n}(\zeta)\right)-T\left(\theta_{k}(\zeta)\right)\right\| & \leq \| S^{-1}\left[u^{\alpha} S\left\{\psi^{2}\left(\theta_{n}(\zeta)-\theta_{k}(\zeta)\right\}\right] \|\right. \\
& +\left\|S^{-1}\left[u^{\alpha} S\left\{-\frac{\beta}{2} \frac{d^{2}}{d \zeta^{2}}\left(\theta_{n}^{2}(\zeta)-\theta_{k}^{2}(\zeta)\right)\right\}\right]\right\|
\end{aligned}
$$

The above can be further be transformed using the property of norm and integral as follows

$$
\begin{aligned}
\left\|T\left(\theta_{n}(\zeta)\right)-T\left(\theta_{k}(\zeta)\right)\right\| & \leq S^{-1}\left[u^{\alpha} S\left\{\left\|\left\{\psi^{2}\left(\theta_{n}(\zeta)-\theta_{k}(\zeta)\right)\right\}\right\|\right\}\right] \\
& +S^{-1}\left[u^{\alpha} S\left\{\left\|\left\{-\frac{\beta}{2} \frac{d^{2}}{d \zeta^{2}}\left(\theta_{n}^{2}(\zeta)-\theta_{k}^{2}(\zeta)\right)\right\}\right\|\right\}\right]
\end{aligned}
$$

Since

$$
\left\|\psi^{2}\left(\theta_{n}(\zeta)-\theta_{k}(\zeta)\right)\right\| \leq \beta_{1}\left\|\theta_{n}(\zeta)-\theta_{k}(\zeta)\right\|
$$

and

$$
\left\|\frac{\beta}{2} \frac{d^{2}}{d \zeta^{2}}\left(\theta_{n}^{2}(\zeta)-\theta_{k}^{2}(\zeta)\right)\right\| \leq \beta_{2} \kappa\left\|\theta_{n}(\zeta)-\theta_{k}(\zeta)\right\|
$$


It implies

$$
\left\|T\left(\theta_{n}(\zeta)\right)-T\left(\theta_{k}(\zeta)\right)\right\| \leq\left\{\beta_{1}-\beta_{2} k\right\}\left\|\theta_{n}(\zeta)-\theta_{k}(\zeta)\right\|
$$

with $\left\{\beta_{1}-\beta_{2} \kappa\right\}<1, \forall \beta_{1} \beta_{2}$. Hence, the nonlinear T-self mapping has a fixed point. This completes the proof.

Further we show that, $T$ satisfies the condition in theorem (2), thus putting

$$
C=0, c=\left\{\beta_{1}-\beta_{2} k\right\}
$$

implies the theorem (1) holds for the nonlinear mapping $T$. Since all condition in previous theorem hold for the defined non-linear mapping $T$. Hence, $T$ is Picards $T$ - stable. This completes the proof.

\section{Result and Discussion}

\subsection{Temperature Distribution}

Here, the governing equation (18) has been solved analytically by using Adomian Decomposition Sumudu Transform Method. When the thermal conductivity is constant, i.e., $\beta=0$, equation (16) becomes a linear differential equation, and the analytical solution for dimensionless temperature distribution $\theta$ is

$$
\theta_{\text {analytical }}=\frac{1}{\left(e^{\psi}+e^{-\psi}\right)}\left(e^{\psi \zeta}+e^{-\psi \zeta}\right)
$$

Figures 2, 4 and 6 discusses the variation of temperature distribution along the fin surface with $\beta$ varying from -0.5 to 0.5 and for different values of $\psi=0.5, \psi=1.0$ and $\psi=1.5$ which shows that the temperature distribution be increases along the coordiantes and the obtained result is very close to the results of Coskun et al. [6] for classical order $\alpha=2.0$. Hence, it provides a good approximation with VIM.

Simillarly, figure 3, 5 and 7 discusses the variation of temperature distribution along the fin surface with $\beta$ varying from -0.5 to 0.5 for different values of $\psi=0.5, \psi=1.0$ and $\psi=1.5$ and for fractional order $\alpha=1.75$ and 1.5 respectively, which shows that, the temperature distribution $\theta$ be increases with fractional order $\alpha$ for different thermal conductivity $\beta$. 


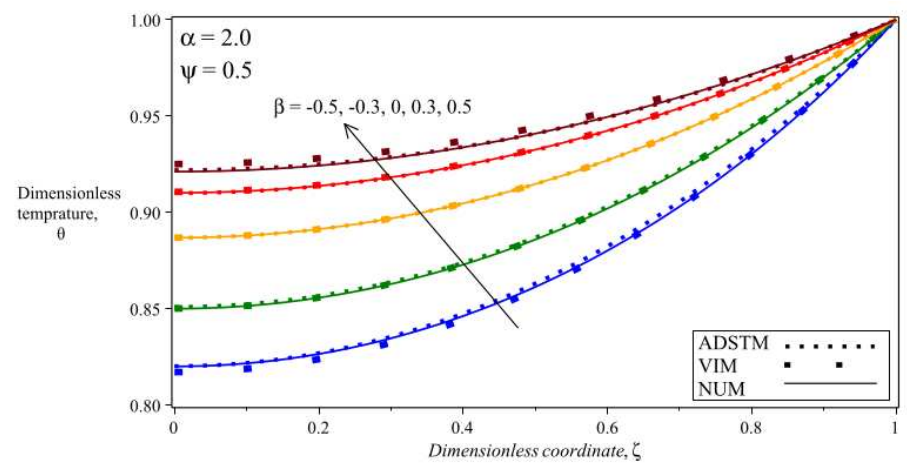

Figure 2: Comparison for temperature distribution within the fin for $\alpha=2.0, \psi=0.5$ and $\beta=-0.5 \ldots 0.5$.

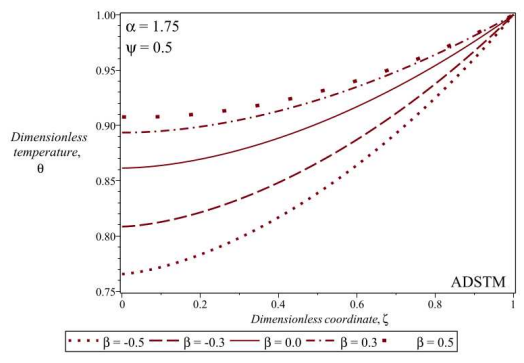

(a)

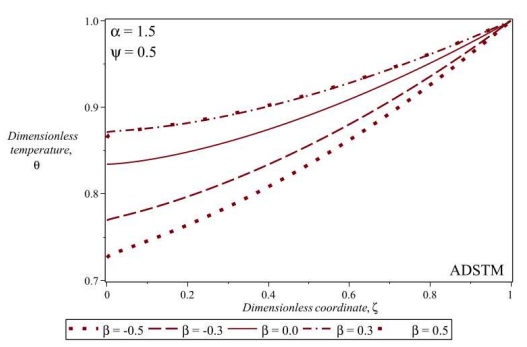

(b)

Figure 3: Variation of dimensionless fin temperature for $\psi=0.5$ and for fractional values (a) $\alpha=1.75$ and (b) $\alpha=1.5$.

\subsection{Fin Efficiency}

The heat transfer rate from the fin can be obtained by using Newtons law of cooling, which is defined by

$$
E=\int_{0}^{b} P\left(T-T_{a}\right) d x
$$

Fin performance can also be characterized by fin efficiency (Kraus et al., 2002).

$$
\eta=\frac{E}{E_{\text {ideal }}}=\frac{\int_{0}^{b} P\left(T-T_{a}\right) d x}{P b\left(T_{b}-T_{a}\right)}=\int_{\zeta=0}^{1} \theta(\zeta) d \zeta
$$




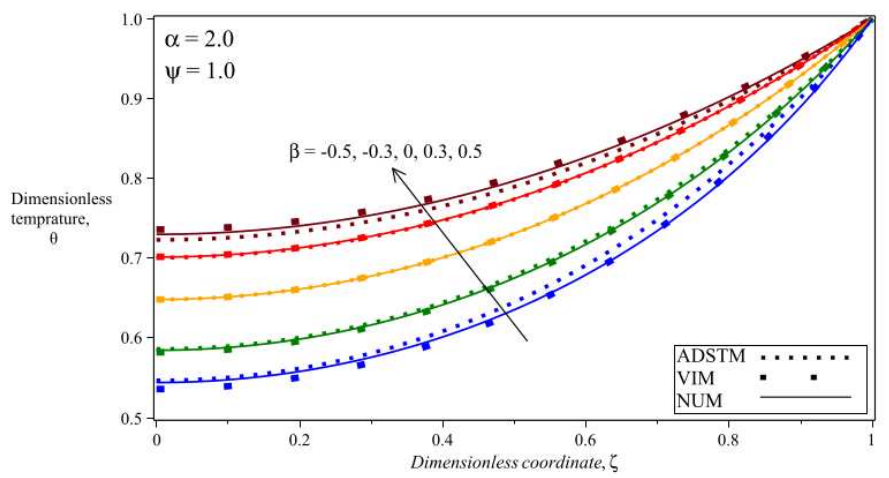

Figure 4: Comparison for dimensionless temperature variation for $\alpha=$ $2.0, \psi=1.0$ and $\beta=-0.5 \ldots 0.5$.

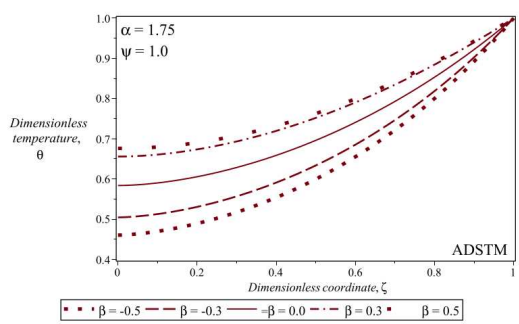

(a)

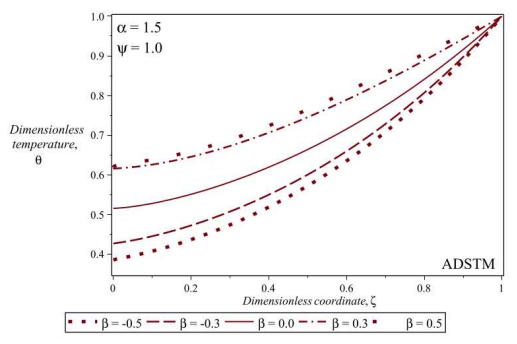

(b)

Figure 5: Variation of dimensionless fin temperature for $\psi=1.0$ and for fractional values (a) $\alpha=1.75$ and (b) $\alpha=1.5$.

On substituting equations (28) into equation (42) and then integrating, the dimensionless fin efficiency for convective straight fins can be obtained.

Figure 8 discusses the variation of fin efficiency for different values of thermal conductivity parameter i.e. $\beta=-0.6,-0.4,-0.2,0.0,0.2,0.4,0.6$ and for classical order $\alpha=2.0$. It shows that there is a minor fluctuation with the results of Coskun et al. [6] for $\beta=0.6$ which can be reduced by taking more number of approximations in the solution. It can be concluded that, with the increase of thermo-geometric fin parameter $(\psi)$, the value of fin efficiency is also increases and it is an important factor that affects the behavior of the solution.

Figure 9 discusses the variation of fin efficiency with thermo-geometric fin 


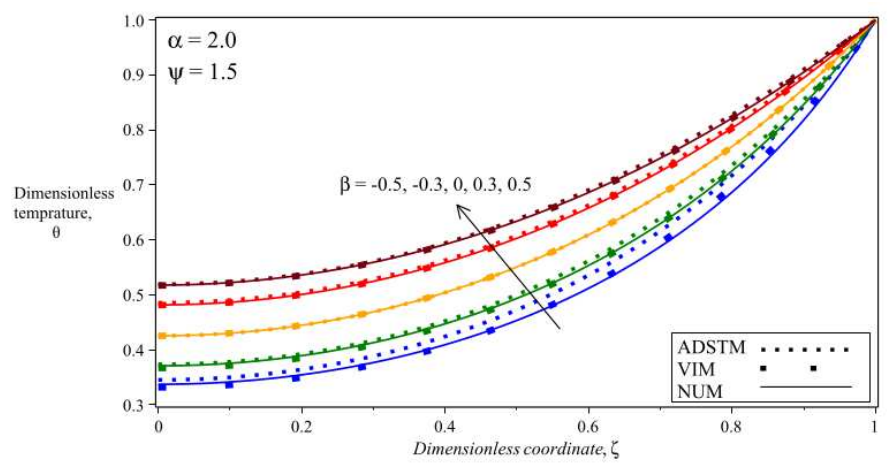

Figure 6: Comparison for dimensionless temperature variation for $\alpha=$ $2.0, \psi=1.5$ and $\beta=-0.5 \ldots 0.5$.

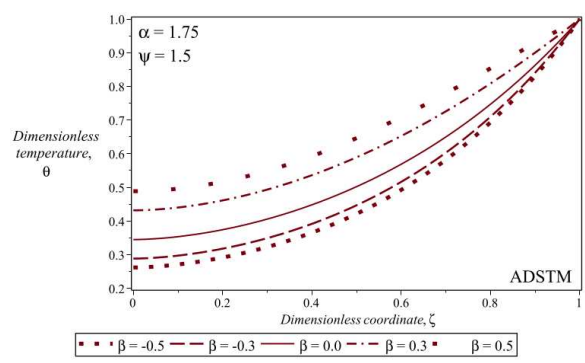

(a)

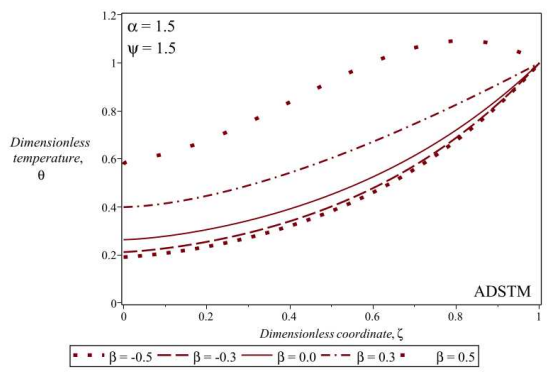

(b)

Figure 7: Variation of dimensionless fin temperature for $\psi=1.5$ and for fractional values (a) $\alpha=1.75$ and (b) $\alpha=1.5$.

parameter, $\psi$ for different values of thermal conductivity parameter, $\beta$ which shows that the fin efficiency is better between the fractional value $\alpha=1.75$ and integer value $\alpha=2$ for $\beta \geq 0$.

\section{Conclusion}

In this work, Adomian decomposition Sumudu transform method is used to study the temperature distribution and fin efficiency in convective straight fins with temperature dependent thermal conductivity for integer as well as fractional values $\alpha$. It is an iterative technique and an effective method for finding 


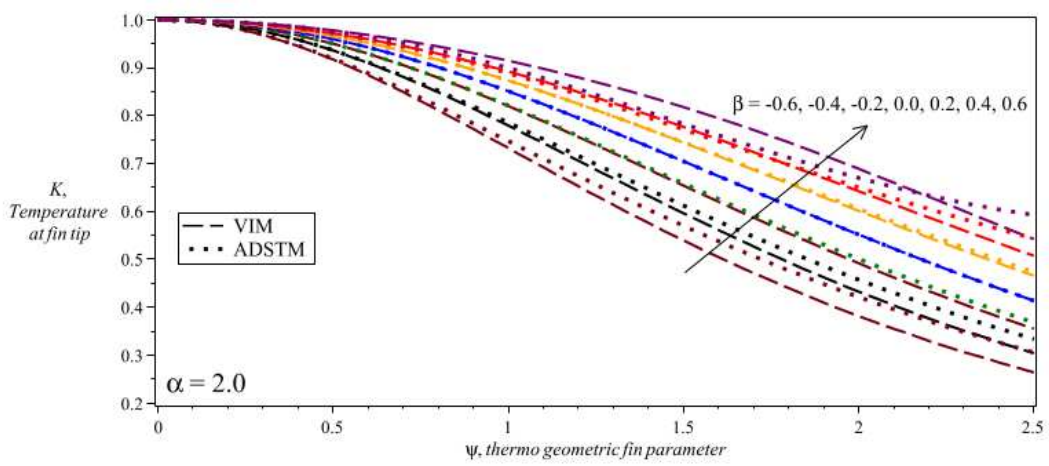

Figure 8: Comparison of fin efficiency with dimensionless parameter $\psi$ and for different values of $\beta$.

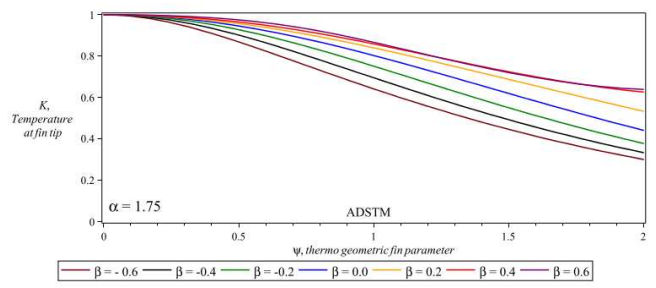

(a)

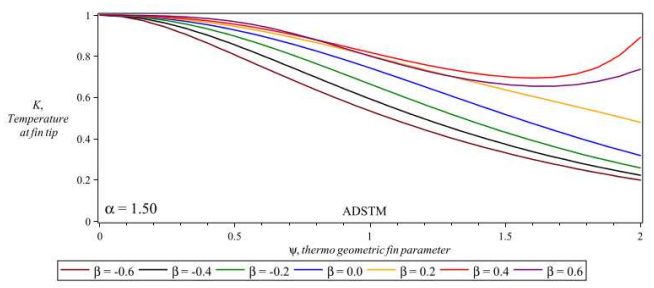

(b)

Figure 9: Variation of fin efficiency with dimensionless parameter $\psi$ for different values of $\beta$ and for fractional values (a) $\alpha=1.75$ and (b) $\alpha=1.5$.

the solution of nonlinear fractional order differential equation where sumudu transform deals with the fractional operator efficiently as compared to other transform like Laplace and Fourier Transform or other integral operator as in Adomian Decomposition method or Homotopy Perturbation method and this is the main advantage of this method. The stability analysis of the proposed method is done by using fixed point theory to check the efficiency of the method. A dimensionless expression for the temperature distribution and fin efficiency has been derived and discussed the effects of thermal conductivity and thermo-geometric fin parameter on temperature distribution which shows that the thermo-geometric fin parameter $(\psi)$ is an important factor that affects the behavior of the solution. The obtained expressions and results may be very 
useful for engineers and scientists in terms of providing a fundamental key while dealing with heat transfer problems in engineering applications.

\section{References}

[1] A.D. Kraus, A. Aziz, J.R. Welty, Extended Surface Heat Transfer, John Wiley and Sons, New York, USA, 2002.

[2] P. Razelos, K. Imre, The optimum dimensions of circular fins with variable thermal parameters, J. Heat Transfer, 102 (1980), 420-425, doi: 10.1115/1.3244316.

[3] M.N. Bouaziz, S. Hanini, Efficiency and optimization of fin with temperature-dependent thermal conductivity: a simplified solution, Heat and Mass Transfer, 44, No. 1 (2007), 1-9, doi: 10.1007/s00231-006-0225-4.

[4] A. Cihat, A decomposition method for fin efficiency of convective straight fins with temperature-dependent thermal conductivity, International Communications in Heat and Mass Transfer, 32 (2005), 831-841, doi: 10.1016/j.icheatmasstransfer.2004.10.006.

[5] A. Rajabi, Homotopy perturbation method for fin efficiency of convective straight fins with temperature-dependent thermal conductivity, Physics Letters A, 364 (2007), 33-37, doi: $10.1016 /$ j.physleta.2006.11.062.

[6] S.B. Coskun, M.T. Atay, Fin efficiency analysis of convective straight fins with temperature dependent thermal conductivity using variational iteration method, Applied Thermal Engineering, 28 (2008), 2345-2352, doi: 10.1016/j.applthermaleng.2008.01.012.

[7] D.D. Ganji, M. Rahimi, M. Rahgoshay, Determining the fin efficiency of convective straight fins with temperature dependent thermal conductivity by using homotopy perturbation method, International Journal of Numerical Methods for Heat $E$ Fluid Flow, 22, No. 2 (2012), 263-272, doi: 10.1108/09615531211199872.

[8] T. Patel, R. Meher, A study on temperature distribution, efficiency and effectiveness of longitudinal porous fins by using Adomian decomposition Sumudu transform method, Procedia Engineering, 127 (2015), 751-758, doi: 10.1016/j.proeng.2015.11.409.

[9] T. Patel, R. Meher, Adomian decomposition Sumudu transform method for convective fin with temperature-dependent internal heat generation and thermal conductivity of fractional order energy balance equation, International Journal of Applied and Computational Mathematics, 2, No. 2 (2016), 7-16, doi: 10.1007/s40819-016-0208-1.

[10] T. Patel, R. Meher, Adomian decomposition sumudu transform method for solving a solid and porous fin with temperature dependent internal heat generation, SpringerPlus, 5, No. 489 (2016), doi: 10.1186/s40064-016-2106-8.

[11] K.S. Miller, B. Ross, An Introduction to the Fractional Calculus and Fractional Differential Equations, John Wiley and Sons, New York, USA, 1993.

[12] I. Podlubny, Fractional Differential Equations, Academic Press, New York, 1999.

[13] H. Eltayeb, A. Kilicman, Application of Sumudu decomposition method to solve nonlinear system of partial differential equations, Abstract and Applied Analysis, 2012, Article ID 412948, 13 pp, doi: 10.1155/2012/412948.

[14] H. Eltayeb, A. Kilicman, M. Said, Application of Sumudu Decomposition Method to Solve Nonlinear System Volterra Integro-differential Equations, Abstract and Applied Analysis, 2014, Article ID 503141, 6 pp., doi: 10.1155/2014/503141. 
[15] G.K. Watugala, Sumudu transform - a new integral transform to solve differential equations and control engineering problems, Mathematical Engineering in Industry, 6 (1998), 319-329, doi: 10.1080/0020739930240105.

[16] Sushila, J. Singh, Y.S. Shishodia, A modified analytical technique for Jeffery-Hamel flow using sumudu transform, Journal of the Association of Arab Universities for Basic and Applied Sciences, 16 (2014), 11-15, doi: 10.1016/j.jaubas.2013.10.001.

[17] M.A. Asiru, Further properties of the Sumudu transformand its applications, International Journal of Mathematical Education in Science and Technology, 33, No. 3 (2002), 441-449, doi: 10.1080/002073902760047940.

[18] F. Belgacem, A. Karaballi, Sumudu transform fundamental properties investigations and applications, Journal of Applied Mathematics and Stochastic Analysis, 2006 (2006), 1-23, doi: $10.1155 / J A M S A / 2006 / 91083$.

[19] A. Kilicman, H. Eltayeb, R.P. Agarwal, On Sumudu Transform and System of Differential Equations, Abstract and Applied Analysis (2010), doi: 10.1155/2010/598702.

[20] A. Kilicman, H. Eltayeb, A note On integral transforms and partial differential equations, Applied Mathematical Sciences, 4, No. 3 (2010), 109-118.

[21] G. Adomian, Solving Frontier Problems in Physics: The Decomposition Method, Kluwer Academic Publishers, Dordrecht, 1988.

[22] A. Atangana, On the new fractional derivative and application to nonlinear Fishers reaction-diffusion equation, Applied Mathematics and Computation, 273 (2016), 948956, doi: 10.1016/j.amc.2015.10.021. 